\title{
Clinical outcomes of EUS-guided drainage of debris-containing pancreatic pseudocysts: a large multicenter study
}

\section{두)(우우}

\section{Authors}

Dennis Yang ${ }^{1}$, Sunil Amin², Susana Gonzalez², Daniel Mullady², Steven A. Edmundowicz ${ }^{4}$, John M. DeWitt ${ }^{5}$, Mouen A. Khashab ${ }^{6}$, Andrew Y. Wang ${ }^{7}$, Satish Nagula ${ }^{2}$, Jonathan M. Buscaglia ${ }^{8}$, Juan Carlos Bucobo ${ }^{8}$, Mihir S. Wagh ${ }^{4}$, Peter V. Draganov ${ }^{1}$, Tyler Stevens ${ }^{9}$, John J. Vargo ${ }^{9}$, Harshit S. Khara ${ }^{10}$, David L. Diehl ${ }^{10}$, Rajesh N. Keswani ${ }^{11}$, Srinadh Komanduri ${ }^{11}$, Patrick S. Yachimski ${ }^{12}$, Anoop Prabhu ${ }^{13}$, Richard S. Kwon ${ }^{13}$, Rabindra R. Watson ${ }^{14}$, Adam J. Goodman ${ }^{15}$, Petros Benias ${ }^{16}$, David L. Carr-Locke ${ }^{16}$, Christopher J. DiMaio $^{2}$

\section{Institutions}

1 Division of Gastroenterology, University of Florida College of Medicine, Gainesville, Florida, United Sates

2 Division of Gastroenterology, Icahn School of Medicine at Mount Sinai, New York, New York, United States

3 Division of Gastroenterology, Washington University School of Medicine, St Louis, Missouri, United States

4 Division of Gastroenterology, University of Colorado, Aurora, Colorado, United States

5 Division of Gastroenterology, Indiana University School of Medicine, Indianapolis, Indiana, United States

6 Division of Gastroenterology and Hepatology, Johns Hopkins Hospital, Baltimore, Maryland, United States

7 Division of Gastroenterology and Hepatology, University of Virginia Health System, Charlottesville, Virginia, United States

8 Division of Gastroenterology and Hepatology, Stony Brook University School of Medicine, Stony Brook, New York, United States

9 Department of Gastroenterology and Hepatology, Digestive Disease Institute, Cleveland Clinic, Cleveland, Ohio, United States

10 Department of Gastroenterology and Nutrition, Geisinger Medical Center, Danville, Pennsylvania, United States

11 Division of Gastroenterology and Hepatology, Northwestern University Feinberg School of Medicine, Chicago, Illinois, United States

12 Division of Gastroenterology, Hepatology and Nutrition, Vanderbilt University Medical Center, Nashville, Tennessee, United States

13 Division of Gastroenterology, University of Michigan Medical School, Ann Arbor, Michigan, United States

14 Division of Digestive Diseases, University of California at Los Angeles School of Medicine, Los Angeles, California, United States

15 Division of Gastroenterology, New York University School of Medicine, New York, New York, United States

16 Division of Digestive Diseases, Beth Israel Medical Center, New York, New York, United States submitted 30.8.2016

accepted after revision $\mathbf{2 . 1 1 . 2 0 1 6}$

Bibliography

DOI http://dx.doi.org/10.1055/s-0042-121666 |

Endoscopy International Open 2017; 05: EE130-EE136

(c) Georg Thieme Verlag KG Stuttgart · New York

ISSN 2364-3722

\section{Corresponding author}

Christopher J. DiMaio, MD, Dr. Henry D. Janowitz Division of Gastroenterology, Icahn School of Medicine at Mount Sinai, One Gustave L. Levy Place, Box 1069, New York, NY 10029

Fax: +1-212-241-7535

Christopher.DiMaio@mountsinai.org

\section{ABSTRACT}

Background and study aims Data on clinical outcomes of endoscopic drainage of debris-free pseudocysts (PDF) versus pseudocysts containing solid debris (PSD) are very limited. The aims of this study were to compare treatment outcomes between patients with PDF vs. PSD undergoing endoscopic ultrasound (EUS)-guided drainage via transmural stents.

Patients and methods Retrospective review of 142 consecutive patients with pseudocysts who underwent EUS-guided transmural drainage (TM) from 2008 to 2014 at 15 academic centers in the United States. Main outcome measures included TM technical success, treatment outcomes (symptomatic and radiologic resolution), need for endoscopic re-intervention at follow-up, and adverse events (AEs).

Results TM was performed in 90 patients with PDF and 52 with PSD. Technical success: PDF 87 (96.7\%) vs. PSD 51 (98.1\%). There was no difference in the rates for endoscopic re-intervention (5.5\% in PDF vs. $11.5 \%$ in PSD; $P=0.33)$ or AEs $(12.2 \%$ in PDF vs. $19.2 \%$ in PSD; $P=0.33$ ). Median long-term follow-up after stent removal was 297 days (interquartile range [IQR]: 59-424 days) for PDF and 326 days (IQR: $180-448$ days) for PSD $(P=0.88)$. There was a higher rate of short-term radiologic resolution of PDF $(45 ; 66.2 \%)$ vs. PSD ( 21 ; $51.2 \%)(\mathrm{OR}=0.30 ; 95 \% \mathrm{Cl}: 0.13-0.72 ; P=0.009)$. There was no difference in long-term symptomatic resolution (PDF: $70.4 \%$ vs. PSD: $66.7 \% ; P=0.72$ ) or radiologic resolution (PDF: $68.9 \%$ vs. PSD: $78.6 \% ; P=0.72$ )

Conclusions There was no difference in need for endoscopic reintervention, AEs or long-term treatment outcomes in patients with PDF vs. PSD undergoing EUS-guided drainage with transmural stents. Based on these results, the presence of solid debris in pancreatic fluid collections does not appear to be associated with a poorer outcome. 


\section{Introduction}

Pancreatic fluid collections (PFCs) can develop as a consequence of pancreatic duct leak/disruption or pancreatic inflammation [1-3]. A pancreatic pseudocyst is a type of PFC enclosed in a non-epithelialized wall. While pseudocysts develop in up to $20 \%$ of cases of acute pancreatitis and $40 \%$ of patients with chronic pancreatitis, most of them resolve spontaneously [4]. Treatment is warranted in the setting of symptoms (i.e. abdominal pain, early satiety, weight loss, luminal and/or biliary obstruction) and/or cyst-associated adverse events (i.e. infection, bleeding) [5].

Endoscopic transmural (TM) drainage has become first-line therapy for symptomatic pancreatic pseudocysts given its similar efficacy, shorter recovery times, fewer adverse events (AEs) and improved cost-effectiveness when compared to surgical cystogastrostomy [6]. This technique involves the creation of a communication between the pseudocyst and the gastroduodenal lumen (cystogastrostomy or cystoduodenostomy), allowing the internal drainage and collapse of the pseudocyst [7].

Several factors have been associated with the technical and clinical success of endoscopic drainage. Presence of solid debris within the cystic cavity has been historically regarded as a negative factor on endoscopic drainage, in part due to the worse clinical outcomes in patients with walled-off necrosis (WON) [8]. The revised Atlanta classification distinguishes different types of PFCs based on morphologic features seen on contrastenhanced computed tomography (CECT) [1]. WON is defined as a heterogeneous collection with varying amounts of non-liquid density that has developed from acute necrotizing pancreatitis whereas a pseudocyst is defined as a well-encapsulated homogenous fluid collection with minimal or no solid debris arising from interstitial edematous pancreatitis. In spite of this categorical distinction, it is not uncommon in clinical practice to encounter some degree of solid debris within a pseudocyst at the time of EUS-guided drainage. However, as opposed to WON, data on clinical outcomes of TM drainage of pseudocysts containing variable amount of solid debris (PSD) are very limited. The primary aim of this multicenter, retrospective study was to compare symptomatic and radiologic resolution rates at followup in patients undergoing EUS-guided drainage of debris-free pseudocysts (PDF) versus PSD. A secondary aim was to assess the need for endoscopic re-intervention and adverse events between the two groups.

\section{Patients and methods}

This multicenter retrospective study included all consecutive patients aged $\geq 18$ years who underwent attempted EUS-guided pseudocyst drainage at 15 academic tertiary referral centers in the United States between January 2008 and September 2014. Patients were identified through prospectively maintained endoscopic databases and chart review. All data were extracted and compiled into a central database. Informed procedural consents were obtained from all patients. This study was approved by the institutional review board for human research at each of the participating institutions. All endoscopic procedures were performed according to the ASGE practice guideline recommendations on antibiotic prophylaxis and management of antithrombotic agents and coagulopathy $[9,10]$.

All patients with clinical and/or radiographic evidence of necrotizing pancreatitis and features of WON on index imaging (based on the revised Atlanta classification) were excluded. All patients who had any other form of intervention (i.e. percutaneous, surgical and/or endoscopic transpapillary drainage) prior to EUS-guided drainage were also excluded.

Data collection was separated into 3 categories: baseline, procedural, and post-procedural data. Baseline data of interest included patient demographics, etiology of pancreatitis, presence of chronic pancreatitis, pseudocyst characteristics, and findings on index imaging prior to drainage. Procedure-related data included technical aspects for TM drainage. Relevant data included presence or absence of solid debris within the pseudocyst, method of cystoenterostomy tract creation, route of drainage, type and number of transmural stent(s) used. All procedure-related AEs were reviewed. Post-procedure data included duration of follow-up, need for additional intervention(s), and treatment outcomes.

\section{Definitions}

PFCs were classified as per the revised Atlanta classification as acute peripancreatic fluid collection (APFC), pseudocysts, acute necrotic collection (ANC) or walled-off necrosis (WON) [9]. AEs were assessed based on previously established criteria by the American Society of Gastrointestinal Endoscopy (ASGE) [11]. Patients were divided into 2 groups: debris-free pseudocyst (PDF) versus pseudocyst with solid debris (PSD); this definition was based on the pseudocyst characteristics found on EUS examination at time of TM drainage. PDF was defined as absence of any debris within a pseudocyst, whereas PSD was defined as a pseudocyst containing variable amounts of solid material ( Fig.1). TM technical success was defined as successful placement of a minimum of one transmural stent during pseudocyst drainage. Symptom resolution was defined as the complete absence of any symptoms, including pain, gastric outlet obstruction, biliary obstruction, and/or infection. Radiologic resolution was defined as the complete resolution of the pseudocyst on repeat imaging at the time of follow-up.

\section{Main outcome measures}

The primary aim of the study was to compare symptomatic and radiologic resolution of patients with PDF vs. PSD who underwent EUS-guided TM drainage. A secondary aim was to compare rates of endoscopic re-intervention and adverse events between the two groups.

\section{Follow-up}

Clinical and radiologic follow-up was performed at the discretion of the endoscopist and care team at each participating institution. Treatment outcome measures were evaluated at both short-term and long-term follow-up. In clinical practice, most TM stents are usually kept in place for a period of 3 weeks to 5 weeks, at which time repeat evaluation (i.e. clinic visit, imaging) is performed in order to assess interval changes and help dictate timing of stent removal. We defined short-term follow- 


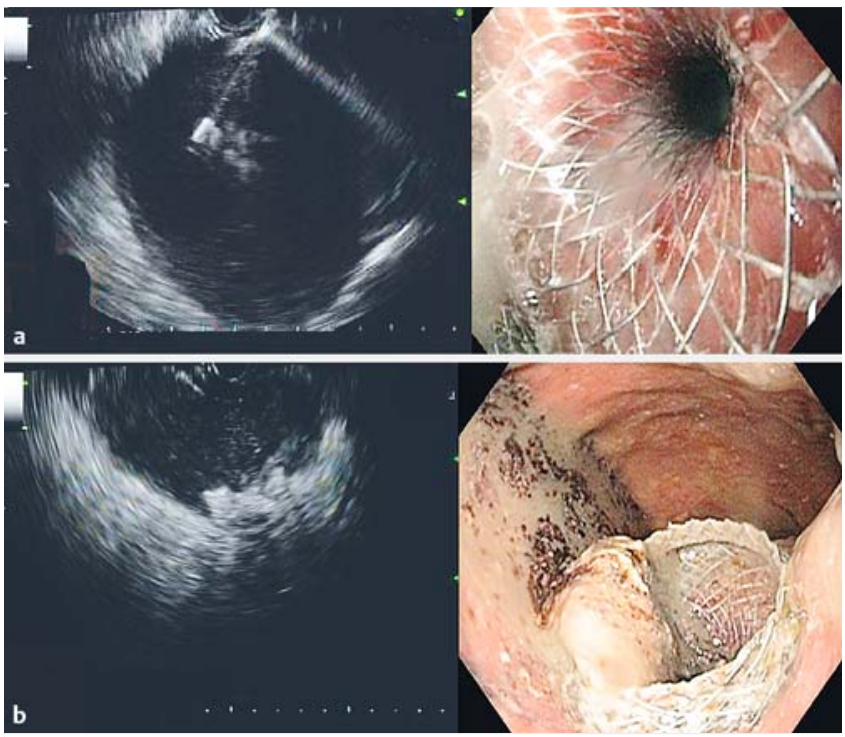

- Fig. 1 a Endosonographic and endoscopic view of EUS-guided transmural drainage of a debris-free pseudocyst. b Endosonographic and endoscopic view of EUS-guided transmural drainage of a pseudocyst containing solid debris.

up as an interval $\geq 2$ weeks following transmural stent placement but prior to stent removal in order to assess treatment efficacy at the time of patient reevaluation. Long-term follow-up was defined as a period $\geq 2$ weeks following transmural stent removal. The main objective of using this definition was to evaluate clinical outcomes once the stent had been removed and not prior to this event.

\section{Statistical analysis}

Descriptive statistics were calculated for all demographic, imaging, and clinical variables and were reported as mean \pm standard deviation (SD), median with interquartile ranges (IQR), and/ or as a proportion. Univariate analysis was performed by using the chi-square test and the Fisher's exact test for categorical variables and Mann-Whitney test for continuous variables when indicated. All statistical analysis was performed with the SPSS software v22 (IBM, SPSS Statistics, Armonk, NY). A P value less than 0.05 was considered statistically significant.

\section{Results}

\section{Patient characteristics and pancreatic pseudocysts}

Two hundred and fifteen patients underwent EUS-guided TM drainage during the study period. A total of 142 patients were diagnosed with pseudocysts based on the revised Atlanta classification [9]. Of these patients, there were 90 patients with PDF and 52 with PSD based on EUS imaging characteristics at the time of TM drainage.

Baseline characteristics of patients with pseudocysts who underwent TM drainage are summarized in $>$ Table 1 . There was no significant differences in patient age, sex, etiology of acute pancreatitis, presence of chronic pancreatitis or pseudocyst location in patients with PDF vs. PSD. The median size of the pseudocysts was similar in both groups: PDF $(9.0 \mathrm{~cm}$; IQR: $7.4-12.2 \mathrm{~cm})$ and PSD $(9.0 \mathrm{~cm}$; IQR: $7.5-13.3 \mathrm{~cm})(P=0.92)$. Overall, abdominal pain was the most common indication for pseudocyst drainage in both groups (90\% in PDF vs. $92.3 \%$ in PSD; $P=0.77)$. Other indications included gastric outlet obstruction, biliary obstruction, and/or infection ( $>$ Table 1 ).

\section{Technical parameters and outcomes}

\section{EUS-guided transmural drainage}

The procedural technique of EUS-guided drainage for patients with PDF vs. PSD is summarized in > Table 2 . In both groups, a 19-gauge EUS needle was used for access and fistula creation via a transgastric or transduodenal approach in most cases. There was no significant difference in the type of transmural stent(s) used for pseudocyst drainage between the two groups. The most common TM drainage intervention was placement of a median of $210 \mathrm{Fr}$ double-pigtail plastic stents (53.3\% in PDF vs. $57.7 \%$ in PSD; $P=0.73$ ) or $27 \mathrm{Fr}$ double-pigtail plastic stents (38.9\% in PDF vs. $30.8 \%$ in PSD; $p=0.37$ ). In all cases where a metal stent was placed for TM drainage ( $\$$ Table 2 ), a $7 \mathrm{Fr}$ or $10 \mathrm{Fr}$ plastic double pigtail stent was placed through the lumen for anchorage and to assist with drainage. Overall, stent(s) remained in place for a median of 97 and 95 days in the PDF and PSD groups, respectively $(P=0.25)$.

\section{Technical success and need for endoscopic re-intervention}

Technical success rate for TM drainage was $96.7 \%$ (87/90) in PDF and $98.1 \%(51 / 52)$ in PSD $(P=0.63)$. Reasons for failure in the 3 patients from the PDF group included inability to dilate the fistulous tract in 1, hypoxemia with termination of the procedure in 1, and failure to advance the stent in 1 patient. TM drainage was unsuccessful in 1 patient with PSD due to stent migration following deployment resulting in perforation. The migrated stent was removed followed by endoscopic closure of the gastric defect.

There was no difference in the need for endoscopic re-intervention (within 4 weeks from time of TM drainage) between patients with PDF (5/90; 5.5\%) vs. PSD (6/52; 11.5\%) (OR 2.22; $95 \% \mathrm{Cl}$ : $0.64-7.66 ; P=0.33)$. Endoscopic re-intervention consisted of stent replacement and/or upsizing. Infection was the most common indication for re-intervention for PDF (2/90; $2.2 \%$ ) and PSD (4/52; $7.7 \%)$ (OR 0.27; $95 \% \mathrm{Cl}: 0.05-1.5 ; P=$ $0.19)$. Persistent or enlarging symptomatic pseudocyst was an indication for re-intervention in 1 patient with PDF and in 2 patients with PSD (OR 0.28; $95 \% \mathrm{Cl}: 0.02-3.18$; $P=0.55)$. Endoscopic re-intervention was performed for stent migration in 2 patients with PDF but none with PSD $(P=0.53)$. There were no cases of stent occlusion requiring revision in either group.

\section{Procedural adverse events}

Overall, AE rates were not significantly different between patients with PDF (12.2\%) vs. PSD (19.2\%) $(P=0.33)$. Procedural AEs for pseudocyst drainage are summarized in > Table 3. Bleeding was managed with endoscopic hemostasis and blood transfusion ( $n=4)$ or coil embolization by interventional radiology $(n=4)$. Three cases of perforation during TM stent place- 
- Table 1 Patient characteristics of patients with pseudocysts undergoing endoscopic drainage.

\begin{tabular}{|c|c|c|c|}
\hline Baseline characteristics & PDF $(n=90)$ & PSD $(n=52)$ & $P$ value \\
\hline Age, mean (SD), years & $53(16.1)$ & $51.9(16.8)$ & 0.54 \\
\hline Males; n(\%) & $61(67.8)$ & $32(61.5)$ & 0.47 \\
\hline $\begin{array}{l}\text { Etiology of acute pancreatitis, n(\%) } \\
\text { ETOH } \\
\text { Gallstones } \\
\text { Idiopathic } \\
\text { Other }\end{array}$ & $\begin{array}{l}27(30) \\
21(23.3) \\
23(25.6) \\
19(21.1)\end{array}$ & $\begin{array}{r}17(32.7) \\
16(30.8) \\
10(19.2) \\
9(17.3)\end{array}$ & $\begin{array}{l}0.85 \\
0.43 \\
0.42 \\
0.67\end{array}$ \\
\hline Presence of underlying chronic pancreatitis, n (\%) & $22(24.4)$ & $13(25)$ & 1.00 \\
\hline Size of drained pseudocyst, median (interquartile range), $\mathrm{cm}$ & $9(7.4-12.2)$ & $9(7.5-13.3)$ & 0.92 \\
\hline $\begin{array}{l}\text { Pseudocyst location; n(\%) } \\
\text { Head } \\
\text { Body } \\
\text { Tail }\end{array}$ & $\begin{array}{l}22(24.4) \\
52(57.8) \\
30(33.3)\end{array}$ & $\begin{array}{l}13(25) \\
35(67.3) \\
20(38.5)\end{array}$ & $\begin{array}{l}1.00 \\
0.29 \\
0.59\end{array}$ \\
\hline $\begin{array}{l}\text { Indication for drainage, } \mathrm{n}(\%) \\
\text { Pain } \\
\text { Gastric outlet obstruction } \\
\text { Biliary obstruction } \\
\text { Infection }\end{array}$ & $\begin{array}{l}81(90) \\
19(21.1) \\
4(4.4) \\
8(8.9)\end{array}$ & $\begin{array}{l}48(92.3) \\
7(13.5) \\
1(1.9) \\
6(11.5)\end{array}$ & $\begin{array}{l}0.77 \\
0.37 \\
0.65 \\
0.77\end{array}$ \\
\hline
\end{tabular}

- Table2 Transmural drainage technique.

\begin{tabular}{|c|c|c|c|c|}
\hline Transmural drainage technique & PDF $(n=90)$ & $\operatorname{PSD}(n=52)$ & OR $(95 \% \mathrm{Cl})$ & $P$ value \\
\hline $\begin{array}{l}\text { Access route; } \mathrm{n}(\%) \\
\text { Trans-gastric } \\
\text { Trans-duodenal } \\
\text { Unspecified }\end{array}$ & $\begin{array}{c}79(87.8) \\
5(5.6) \\
6(6.7)\end{array}$ & $\begin{array}{c}45(86.5) \\
5(9.6) \\
2(3.8)\end{array}$ & $\begin{array}{l}1.12(0.40-3.09) \\
0.55(0.15-2.01) \\
1.79(0.35-9.19)\end{array}$ & $\begin{array}{l}1.00 \\
0.50 \\
0.71\end{array}$ \\
\hline $\begin{array}{l}\text { Type of transmural stent placed, } \mathrm{n}(\%) \\
\text { Plastic } \\
\text { Metal } \\
\text { Lumen-apposing transmural stent } \\
\text { Unspecified }\end{array}$ & $\begin{array}{l}72(80) \\
10(11.1) \\
2(2.2) \\
6(6.7)\end{array}$ & $\begin{array}{l}37(71.2) \\
12(23.1) \\
3(5.8) \\
0\end{array}$ & $\begin{array}{l}1.62(0.73-3.58) \\
0.42(0.17-1.05) \\
0.37(0.06-2.30) \\
-\end{array}$ & $\begin{array}{l}0.30 \\
0.09 \\
0.36 \\
0.08\end{array}$ \\
\hline Endoscopic re-intervention within 4 weeks & $5(5.5)$ & $6(11.5)$ & $0.45(0.13-1.56)$ & 0.33 \\
\hline
\end{tabular}

- Table3 Adverse events.

\begin{tabular}{|l|c|c|c|c|}
\hline Adverse event & PDF (n=90) & PSD (n= 52) & OR (95\% Cl) & P value \\
\hline Bleeding & $4(4.4)$ & $4(7.7)$ & $0.79(0.43-7.49)$ & 0.46 \\
\hline Perforation & $2(2.2)$ & $2(3.8)$ & $1.76(0.24-12.88)$ & 0.62 \\
\hline Infection & $2(2.2)$ & $4(7.7)$ & $0.27(0.05-1.5)$ & 0.19 \\
\hline Cardiopulmonary & $1(1.1)$ & 0 & - & 1.00 \\
\hline Death & $1(1.1)$ & 0 & - & 1.00 \\
\hline Other & $1(1.1)$ & 0 & $0.58(0.23-1.49)$ & 1.00 \\
\hline Overall & $11 / 90(12.2)$ & $10 / 52(19.2)$ & 0.33 \\
\hline
\end{tabular}

ment did not require any specific intervention whereas endoscopic removal of a migrated stent and gastric defect closure was performed in one patient with PSD. Infection was reported in 6 patients ( 2 with PDF and 4 with PSD; $P=0.19$ ). All of these patients were managed with antibiotic therapy and repeat endoscopic drainage with upsizing of TM stent(s). In addition to antibiotics and stent upsizing, 4 of these 6 patients also had additional interventions: 2 patients ( 1 with PDF and 1 with PSD) had concomitant insertion of a nasocystic drain and 2 patients (both with PSD) underwent computed tomography-guided 
percutaneous drain placement. These adjunct interventions were performed given concern that the infected pseudocyst would not be adequately drained by TM stent upsizing alone. One patient with PDF died from cardiopulmonary arrest following aspiration.

\section{Treatment outcomes and follow-up}

The median short-term follow-up was the same for patients with PDF (50 days; IQR: 35 - 86 days) vs. PSD (50 days; IQR: $37-75$ days) $(P=0.87)$. Complete symptomatic resolution at short-term follow-up was reported in 39 (70.9\%) patients with PDF and 25 (58.1\%) patients with PSD (Odds ratio [OR] = 1.56; $95 \% \mathrm{Cl}: 0.66-3.67 ; P=0.38$ ) ( $\mathbf{F i g . 2}$ ). Radiologic resolution of PFC was significantly higher in PDF group (45/68; 66.2\%) compared to the PSD group $(21 / 41 ; 51.2 \%)(\mathrm{OR}=0.30 ; 95 \%$ Cl: $0.13-0.72 ; P=0.009$ ) ( Fig. 2). There was no difference in symptomatic or radiologic resolution rates in neither group (PDF or PSD) between patients who had TM drainage with metal versus plastic stents (data not shown).

The median long-term follow-up was 297 (IQR: 59-424) days for patients with PDF and 326 (IQR: 180-448) days for PSD $(P=0.88)$. At long-term follow-up, there was no significant difference in symptomatic resolution (70.4\% in PDF vs $66.7 \%$ in $\mathrm{PSD})(\mathrm{OR}=0.84 ; 95 \% \mathrm{Cl}: 0.22-3.26 ; P=1.00)$ or radiologic resolution of pseudocyst (68.9\% in PDF vs $78.6 \%$ in PSD) (OR= 1.65; $95 \% \mathrm{Cl}: 0.37-7.39 ; P=0.72$ ) between the 3 cohorts ( Fig. 2). In aggregate, 33 (23.2\%) and 98 (69\%) patients were lost to short- and long-term follow-up, respectively.

\section{Discussion}

Endoscopic TM drainage has largely replaced surgery as firstline therapy for management of pancreatic pseudocysts due its comparable clinical efficacy, shorter post-procedural recovery time, lower costs, and AE rates [6]. While EUS-guided drainage has been recognized as the standard of care for management of symptomatic PFCs $[12,13]$, the optimal endoscopic approach for each type of PFC has not been well defined, particularly in patients with pseudocysts who are subsequently noted to have varying degrees of non-liquid component at the time of the endoscopic procedure (but not seen at time of CECT). In this large multicenter study, there was no difference in longterm treatment outcomes, need for endoscopic re-intervention, or AE rates in patients with debris-free pseudocysts (PDF) versus those with pseudocysts containing solid-debris (PSD) undergoing EUS-guided drainage via transmural stents only.

The Revised Atlanta classification [1] distinguishes types of PFCs based on features on cross-sectional imaging, including whether there is an encapsulating wall and the presence of solid components within the collection. While most pseudocysts are characterized by a well-defined inflammatory capsule with minimal or no solid material, it is not uncommon in clinical practice to identify varying amounts of layering internal debris within the pseudocyst at the time of endoscopic drainage. In cases where viscous fluid and/or layering debris within a pseudocyst may potentially hinder endoscopic drainage, the use of a nasocystic drain in addition to endoprosthesis has been pre-

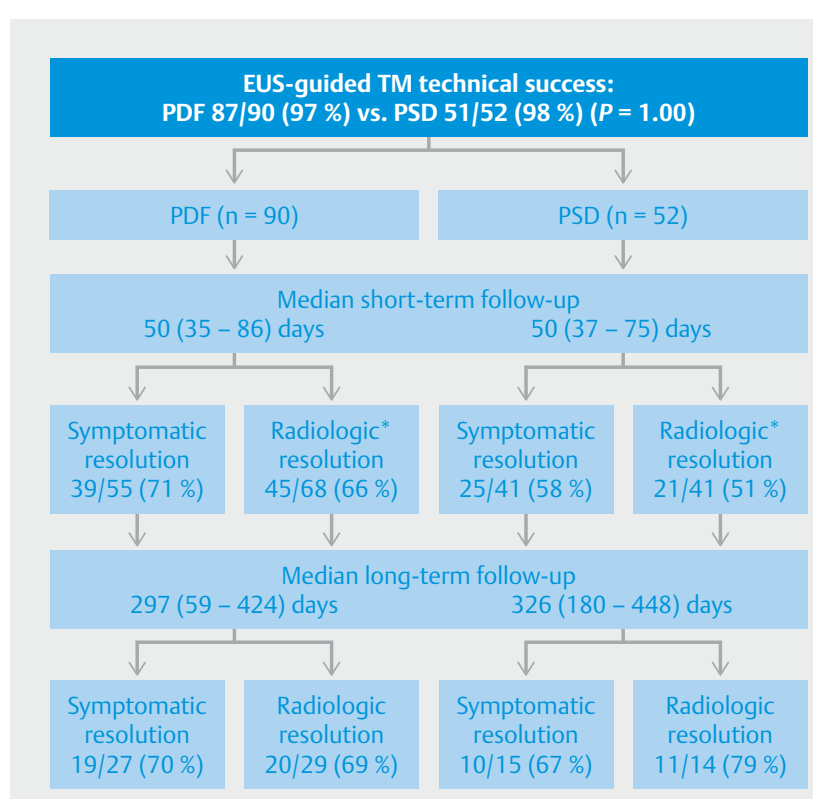

- Fig. 2 Treatment outcomes (symptomatic and radiologic resolution) and follow-up.

viously proposed. In a prospective study of 40 patients, Puri et al demonstrated that pancreatic pseudocyst drainage with both endoprosthesis and nasocystic tube placement was safe and associated with high success rate (32/40; $80 \%)$ [14]. More recently, Siddiqui et al compared outcomes of EUS-guided therapy of PSD by using combined endoprosthesis and a nasocystic drain $(n=63)$ versus transmural stents only $(n=24)$ [15]. Overall, technical success was achieved in $87 / 88$ (99\%) and procedure-related AEs occurred in $9 / 87$ (10.3\%) of the patients. At 1 -month follow-up, relief of symptoms and at least $30 \%$ decrease in pseudocyst size was achieved in $85 \%$ of PSD drained via the combined approach versus $63 \%$ of those treated via stents alone (OR 3.6; $95 \% \mathrm{Cl}: 1.2-10.7 ; P=0.03)$. At a median follow-up of 16 months (range 13-27 months), there was not a significant difference in treatment success rate between patients who had combined drainage (79\%) versus stent alone (58\%) $(P=0.06)$. Overall, the authors concluded that shortterm clinical outcomes in patients with PSD are improved by combined nasocystic drain and endoprosthesis drainage.

There are several potential limitations of adopting a routine combined endoprosthesis and nasocystic drain approach for the management of PSD as suggested by Siddiqui and colleagues. First, all patients in their study were admitted to the hospital after the procedure and pseudocyst lavage via the nasocystic tube was performed with normal saline solution with $100 \mathrm{ml} /$ hour for 48 to 72 hours. Besides the clear risks associated with potential nosocomial exposure during routine postprocedural hospital admission, this clinical practice can significantly increase costs and length of stay associated with the procedure; and thereby, potentially obviates some of the established advantages of endoscopic drainage over a surgical approach. Aggressive irrigation of the cystic cavity requires close monitoring and ancillary staff resources to minimize the risk of 
serious adverse events such as aspiration. Last but not least, many patients may not tolerate an indwelling nasocystic tube for such an extended period of time. As such, it is difficult to advocate for routine use of combined endoprosthesis and nasocystic drain placement in all patients with PSD, especially in the absence of conclusive literature supporting its use in this setting.

In this multicenter study, we demonstrate similar long-term outcomes in patients with PDF versus PSD undergoing EUSguided endoscopic drainage via transmural stents only. Our technical success rate of TM drainage was high and not significantly different for PDF (96.7\%) versus PSD (98.1\%) ( $P=0.63)$. This high technical success rate is comparable and in agreement to those previously reported, alluding to the external validity of our findings $[6-8,13]$. Similar to the prior study by Siddiqui et al, our results suggest higher rate of complete radiologic resolution at short-term follow-up in patients with PDF (66.2\%) compared to PSD (51.2\%) (OR 0.30; $95 \% \mathrm{Cl}: 0.13-$ $0.72 ; P=0.009)$. This would intuitively make sense as a cyst cavity containing layering debris would require more time for its components to completely liquefy and drain when compared to a pseudocyst without any debris. In spite of this radiographic difference, short-term symptomatic resolution rates were similar between both groups and there was also no difference in the need for endoscopic re-intervention (5.5\% in PDF vs. $11.5 \%$ in PSD; $P=0.33$ ) at follow-up. Overall, these findings suggest that while short-term radiologic resolution of pseudocyst may be slower for those with PSD vs. PDF, there was no difference in other surrogate markers (i.e. symptomatic resolution and need for re-intervention) of clinical improvement between the 2 groups. Even more importantly, the results from this study did not demonstrate any statistically significant differences in long-term clinical outcomes or rate of AEs in patients with PDF or PSD undergoing EUS-guided TM drainage.

This study has several strengths. This is the largest and only multicenter experience comparing outcomes in patients with PDF versus PSD undergoing EUS-guided drainage with transmural stents only. Second, all patients with pseudocysts were identified based on established criteria and by the omission of patients with evidence of necrotizing pancreatitis or obvious WON on index cross-sectional imaging in an effort to avoid PFC misclassification [1, 16]. Third, instead of using post-drainage PFC size as the only surrogate marker for treatment success, the main outcomes in this study were defined as complete resolution of symptoms and radiographic findings on follow-up and the need for endoscopic re-intervention, presumably clinically more relevant endpoints. Lastly, not only was treatment efficacy assessed immediately following endoscopic drainage, but long-term treatment effect was estimated by evaluating clinical outcomes after transmural stent removal. This is a key point as ultimate therapeutic outcomes should be assessed once the prostheses are removed, thus signaling the true completion of therapy. $T$

There are also limitations to this study. The study design was retrospective with its inherent limitations. As such, presence of solid debris within a pseudocyst was qualitatively determined by the endoscopist at the time of the EUS but a standardized quantitative assessment was not available across all participating institutions. This potentially increases the heterogeneity within the PSD group and underscores the need for future prospective comparative trials. Second, in aggregate, rates of symptom and radiologic resolution $(68.9 \%-78.6 \%)$ of pseudocysts following endoscopic drainage in this study appear to be lower than those reported in the literature, which range from $75 \%$ to $100 \%[6-8,13,14]$. This discrepancy in outcomes can be in part accounted for by the heterogeneous criteria used in defining treatment success, varied procedural techniques employed, diverse patient populations and timing of the interventions among the prior studies, as well as our own use of very stringent criteria for ST and LT follow-up and definitions of success. Another major limitation is that a significant portion of patients were lost to follow-up and thus the potential for selection bias. Most of the participating institutions in this study are tertiary referral centers and thus a large proportion of patients included in this study were lost to follow-up upon completion of the intervention. We acknowledge that the loss to LT followup limits the interpretability of our current findings and conclusions. Consequently, due to the relative small sample size, the study could have been underpowered to detect statistically significant differences between the 2 groups. Lastly, while this study represents the largest multicenter series evaluating outcomes associated with EUS-guided drainage of PSD, small sample size on subgroup analysis could have precluded detection of any meaningful differences in outcomes. Hence, the lack of statistically significant difference may not necessarily exclude the possibility of clinically relevant differences.

\section{Conclusion}

In summary, this study demonstrated similar treatment outcomes in patients with PDF versus PSD undergoing EUS-guided drainage via placement of transmural stents only. Drainage was successful in most of the patients with placement of transmural plastic stents, which suggests that this may still be an efficacious and cost-effective alternative to use of fully-covered lumen-apposing stents. While short-term radiologic resolution of pseudocyst was higher in PDF versus PSD, there were no differences in need for endoscopic re-intervention or long-term symptomatic or radiologic outcomes between the 2 groups. Future larger comparative prospective studies are needed to evaluate whether transmural stenting alone and the type of stent for that matter, is sufficient for adequate drainage of patients with PSD.

\section{Competing interests}

Dr. Wang receives research funding from Cook Medical. Dr. Edmundowicz is a consultant for Olympus America, Boston Scientific and receives research funding from Boston Scientific. Dr. Khashab is a consultant for Boston Scientific. Dr. Yachimski is a consultant for Boston Scientific. Dr. Keswani is a consultant for Boston Scientific and Cook Medical. Dr. Vargo is a consultant for 
Covidien. Dr. Stevens is a consultant for Boston Scientific. Dr. DiMaio is a consultant for Boston Scientific.

\section{References}

[1] Banks PA, Bollen TL, Dervenis $C$ et al. Classification of acute pancreatitis - 2012: revision of the Atlanta classification and definitions by international consensus. Gut 2013; 62: 102-111

[2] Baillie J. Pancreatic pseudocysts (part I). Gastrointest Endosc 2004; 59: $873-879$

[3] Kozerak RA. Endoscopic drainage of pancreatic pseudocysts. J Hepatobiliary Pancreat Surg 1997; 4: 36-43

[4] Cui ML, Kim KH, Kim HG et al. Incidence, risk factors and clinical course of pancreatic fluid collections in acute pancreatitis. Dig Dis Sci 2014; 59: $1055-1062$

[5] Holt BA, Varadarajulu S. The endoscopic management of pancreatic pseudocysts (with videos). Gastrointest Endosc 2015; 81: 804-812

[6] Varadarajulu S, Bang JY, Sutton BS et al. Equal efficacy of endoscopic and surgical cystogastrostomy for pancreatic pseudocyst drainage in a randomized trial. Gastroenterology 2013; 145: $583-590$

[7] Varadarajulu S, Rana SS, Bhasin DK. Endoscopic Therapy for Pancreatic Duct Leaks and Disruptions. Gastrointest Endosc Clin N Am 2013; 23: $863-892$

[8] Bang JY, Varadarajulu S. Endoscopic ultrasound-guided management of pancreatic pseudocysts and walled-off necrosis. Clin Endosc 2014; 47: $429-431$
[9] Khashab MA, Chithadi KV. ASGE Standards of Practice Committee. et al. Antibiotic prophylaxis for Gl endoscopy. Gastrointest Endosc 2015; 81: $81-89$

[10] Anderson MA, Ben-Menachem T. ASGE Standards of Practice Committee. et al. Management of antithrombotic agents for endoscopic procedures. Gastrointest Endosc 2009; 70: 1060 - 1070

[11] Cotton PB, Eisen GM, Aabakken L et al. A lexicon for endoscopic adverse events: report of an ASGE workshop. Gastrointest Endosc 2010; 71: $450-454$

[12] Sharaiha RZ, DeFilippis EM, Kedia P et al. Metal versus plastic for pancreatic pseudocyst drainage: clinical outcomes and success. Gastrointest Endosc 2015; 82: 822 - 27

[13] Siddiqui AA, Adler DG, Nieto J et al. EUS-guided drainage of peripancreatic fluid collections and necrosis using a novel lumen-apposing stent: a large retrospective multicenter U.S. experience (with videos). Gastrointest Endosc 2015: [Epub ahead of print]; DOI: 10.1016/j. gie.2015.10.020

[14] Puri R, Mishra SR, Thandassery RB et al. Outcome and complications of endoscopic ultrasound guided pancreatic pseudocyst drainage using combined endoprosthesis and naso-cystic drain. J Gastroenterol Hepatol 2012; 27: $722-727$

[15] Siddiqui AA, DeWitt JM, Strongin A et al. Outcomes of EUS-guided drainage of debris-containing pancreatic pseudocysts by using combined endoprosthesis and a nasocystic drain. Gastrointest Endosc 2013; 78: 589- 595

[16] Holt BA, Varadarajulu S. The endoscopic management of pancreatic pseudocysts (with videos). Gastrointest Endosc 2015; 4: 804-812 